\title{
Energetic and Dynamical Aspects of Proton Transfer Reactions in Solution
}

\author{
By R. A. Marcus \\ Department of Chemistry, University of Illinois, Urbana, Illinois 61801 USA
}

Received 19th May, 1975

\begin{abstract}
Several energetic and dynamical aspects of proton transfers are treated. The effect of intrinsic barrier asymmetry on BEBO calculated Brönsted plots is investigated, and contributions to work terms are also considered. The dynamics of transfer of a light particle between two heavier ones is discussed for a particular potential energy surface, making use of classical trajectories, semiclassical concepts, and a previous quantum study. The question of nonequilibrium polarization of solvent is also considered.
\end{abstract}

\section{INTRODUCTION}

It is a pleasure to participate in this symposium honouring Professor R. P. Bell, whose work has illuminated so many parts of the proton transfer field. In this paper I would like to comment on several aspects of proton transfer, both energetic and dynamic : (1) effect of " intrinsic barrier asymmetry" on Brönsted plots, (2) dynamics revealed by recent classical and quantum mechanical studies for an $\mathbf{H}$-atom transfer, (3) contributions to the " work terms, " and (4) the possibility, as in electron transfers, of nonequilibrium polarization of the solvents.

\section{INTRINSIC BARRIER ASYMMETRY AND BRONSTED SLOPES}

Sometime ago we considered a model of a proton transfer reaction, ${ }^{1}$

$$
\mathrm{A}_{1} \mathrm{H}+\mathrm{A}_{2} \rightarrow \mathrm{A}_{1}+\mathrm{HA}_{2}
$$

(charges are omitted for notational brevity), in which the process occurred in three steps :

$$
\begin{aligned}
& A_{1} H+A_{2} \rightarrow A_{1} H \cdots A_{2} \\
& A_{1} H \cdot A_{2} \rightarrow A_{1} \cdots A_{2} \\
& A_{1} \cdots A_{2} \rightarrow A_{1}+H_{2} A_{2} .
\end{aligned}
$$

Of these only the middle one depended on the standard free energy of reaction $\Delta G^{\circ}$ for (2.1)**

Step (2.2) involves a free energy change $w^{\mathbf{r}}$ (called a "work term ") for bringing the reactants close together; $w^{\mathbf{r}}$ includes steric (orientation) effects and, where necessary, ${ }^{2}$ partial desolvation. The next step (2.3) is the actual proton transfer and involves intramolecular and solvent reorganization to form the transition state, followed by an intramolecular and solvent relaxation. Step (2.4) is a " disorienting" and resolvating one; it contains a work term $-w^{\mathfrak{p}}, w^{\mathfrak{p}}$ being the analogue of $w^{\mathrm{x}}$ for the reverse reaction.

* $\Delta G^{\circ \prime}$ is actually the " standard " Gibbs free energy change in the prevailing medium and at the prevailing temperature. 
In an approximation which was quadratic for treating (2.3) and which at the same time neglected " $\lambda$-asymmetry" $\left(\lambda_{1}-\lambda_{2}\right.$, defined later $)$, the rate constant was given by

$$
k_{\mathrm{r}}=Z \exp \left(-\Delta G^{*} / \boldsymbol{k} T\right) \text {, }
$$

apart from the usual statistical factors. ${ }^{*} Z$ is the collision frequency in solution, $\Delta G^{*}$ is

$$
\Delta G^{*}=w^{\mathrm{r}}+(\lambda / 4)\left(1+\Delta G_{\mathrm{R}}^{\circ \prime} / \lambda\right)^{2} \quad\left(\left|\Delta G_{\mathrm{R}}^{\sigma^{\prime}}\right| \leqslant \lambda\right)
$$

and

$$
\Delta G_{\mathrm{R}}^{\circ \prime}=\Delta G^{\mathrm{c \prime}}+w^{\mathrm{p}}-w^{\mathrm{r}} .
$$

$i$ is the "intrinsic" free energy barrier, ${ }^{1}$ i.e., the barrier in (2.3) when $\Delta G_{\mathrm{R}}^{\circ \prime}=0$. $\Delta G_{\mathrm{R}}^{\circ}$ is seen from eqn (2.7) to be the effective standard free energy of reaction for the proton transfer step itself.

Similarly, in a bond energy-bond order (BEBO) type of calculation, the corresponding value of $\Delta G^{*}$ is given ${ }^{1}$ by eqn (2.8) when $i$-asymmetry is neglected and when the $E$ 's in ref. (1) are replaced by free energies,

$$
\Delta G^{*}=w^{\mathrm{r}}+(\lambda / 4)+\left(\Delta G_{\mathrm{R}}^{\circ} / 2\right)+(\lambda / 4 \gamma) \ln \cosh \left(2 \gamma \Delta G_{\mathrm{R}}^{\circ} / \lambda\right)
$$

where $\gamma$ is $\ln 2$.

The difference between eqn (2.6) and (2.8) was typically relatively small. ${ }^{1}$ Implications of the equations are apparent : a small $\lambda$ implies a large curvature of a Brönsted plot; a small $\lambda$ also implies a large limiting rate at large negative $\Delta G_{\mathrm{R}}^{\circ}$ when $w^{\mathrm{r}}$ is small, but the limiting rate is small when $w^{\mathbf{r}}$ is large.

A question which arises is the effect of $\lambda$-asymmetry. Specifically, if a potential energy surface is varied by varying $\Delta G^{\circ \prime}$ of reaction (2.1), holding constant the intrinsic barriers of the exchange reactions

$$
\mathrm{A}_{i} \mathrm{H}+\mathrm{A}_{i} \rightarrow \mathrm{A}_{i}+\mathrm{HA}_{i}, \quad(i=1,2)
$$

do the preceding considerations prevail ${ }^{3}$ The intrinsic barrier $\lambda_{i} / 4$ for the reaction in eqn (2.9) may depend on $i$, and the difference in $\lambda_{1}-\lambda_{2}$ is called here the $\lambda$-asymmetry Differences in $\lambda_{1}$ and $\lambda_{2}$ were neglected in deriving eqn (2.6), prompted in part by a finding that such effects were relatively minor in the quadratic case, i.e., in eqn (2.6). ${ }^{4}$ We consider now their effect on the BEBO derived formula, eqn (2.8).

The problem is how to calculate the effect of varying $\Delta G^{\circ \prime}$ holding the intrinsic barriers constant and not assuming $\lambda_{1}=\lambda_{2}$. Typically, a potential energy surface is not automatically characterized in terms of $\lambda_{1}, \lambda_{2}$ and $\Delta G_{\mathrm{R}}^{\circ \prime}$. For example, in a BEBO model $^{5}$ for the reaction in eqn (2.9), the potential energy of formation of an intermediate state can be written as

$$
\Delta E=V_{1}-V_{1} n_{1}^{p_{1}}-V_{2} n_{2}^{p_{2}},
$$

where, along the reaction path, bond order is conserved:

$$
n_{1}+n_{2} \equiv 1
$$

$n_{i}$ and $V_{i}$ are the bond order and bond energy of the $\mathrm{A}_{i} \mathrm{H}$ 'th bond, $p_{i}$ is an exponent which reflects a property of that bond. For the exchange reaction in eqn (2.9), $n_{i}$ is $\frac{1}{2}$ in the transition state, and so $\Delta E$ for that reaction, which we may call $\Delta E_{1}$, or better yet, $\lambda_{i} / 4$, is found from eqn $(2.10)$ to be $V_{i}\left[1-2\left(\frac{1}{2}\right)^{p i}\right]$.

* If $s^{r}$ and $s^{p}$ are the statistical factors for forward and reverse steps. it suffices to replace $w^{\mathrm{r}}$ and $w^{\mathrm{p}}$ by $w^{\mathrm{r}}-k T \ln s^{\mathrm{r}}, w^{\mathrm{p}}-k T \ln s^{\mathrm{p}}$, to include their effect. ${ }^{1}$ Further, $k_{5}$ is the $k$ in footnote 3 of ref (1) in the case of diffusion effects. 
The potential energy change accompanying the reaction in eqn (2.3) is $\Delta V$ :

$$
\Delta V=V_{1}-V_{2} \text {. }
$$

Thus, the effect of $\Delta V$ on the potential energy barrier to reaction $\Delta E$ can be investigated, holding the intrinsic barriers $\lambda_{1}$ and $\lambda_{2}$ constant, only by varying $p_{1}$ and/or $p_{2}$ simultaneously. (It is not clear that this precaution was followed previously.) ${ }^{3}$ The value of $n_{1}$ in the transition state is obtained by setting $\partial \Delta E / \partial n_{1}=0$ and introducing the resulting $n_{1}$ and $n_{2}$ into eqn (2.10).

Investigation of the effect of $\Delta V$ on $\Delta E$, holding $\lambda_{1}$ and $\lambda_{2}$ constant, is considerably simplified, as in eqn (10) of ref. (1), by noting that $p_{i} \cong 1$ and expanding $n_{i}^{p i}$ in eqn (2.10) in a Taylor series, retaining only the first two terms. The barrier $\Delta E$ is found (eqn (12) of ref. (1)) to be

$$
\Delta E=n_{2}^{\ddagger} \Delta V-\sum_{i=1,2}\left(\lambda_{i} / 4_{j}\right) n_{i}^{\ddagger} \ln n_{i}^{\ddagger},
$$

where $n_{1}^{\ddagger}$ and $n_{2}^{\ddagger}$ are the solution of $\partial \Delta E / \partial n_{1}^{\ddagger}=0$, i.e., of

$$
\left.\begin{array}{l}
0=-\Delta V-\left(\lambda_{1} / 4 \gamma\right)\left(\ln n_{1}^{\ddagger}+1\right)+\left(\lambda_{2} / 4 \gamma\right)\left(\ln n_{2}^{\ddagger}+1\right) \\
n_{1}^{\ddagger}+n_{2}^{\ddagger}=1 .
\end{array}\right\}
$$

(Eqn (2.8) can be obtained from eqn (2.13) and (2.14) by setting $\lambda_{1}=\lambda_{2} \equiv \lambda$, replacing $\Delta V$ by $\Delta G_{R^{\prime}}^{\circ}$, and adding to (2.13) the barrier $w^{\text {r }}$ of the first step (2.2).) Now, at last, $\Delta E$ depends only on $\Delta G_{R}^{\circ}, \lambda_{1}$ and $\lambda_{2}$.

The slope of a $(\Delta E, \Delta V)$ plot at a given $\lambda_{1}$ and $\lambda_{2}$ is obtained by observing that $\mathrm{d} \Delta E / \mathrm{d} \Delta V$ is the sum of $\left(\partial \Delta E / \partial \Delta V_{n_{i}^{\ddagger}}\right)$ and of $\left(\partial \Delta E / \partial n_{1}^{\ddagger}\right)_{\Delta V}\left(\partial n_{1}^{\ddagger} / \partial \Delta V\right)$. Since $\left(\partial \Delta E / \partial n_{1}^{\ddagger}\right)_{\Delta V}$ is zero, one finds from eqn (2.13) that

$$
\partial \Delta E / \partial \Delta V=n_{2}^{\ddagger} \text {. }
$$

\begin{tabular}{|c|c|c|c|c|c|}
\hline$n_{2}^{\ddagger}$ & $\Delta V$ & $\Delta E$ & $n_{2}^{\ddagger}$ & $\Delta V$ & $\Delta E$ \\
\hline 0.1 & -19.2 & 0.4 & 0.6 & 0.0 & 7.2 \\
\hline 0.2 & -15.2 & 1.0 & 0.7 & 5.4 & 10.7 \\
\hline 0.3 & -11.7 & 1.8 & 0.8 & 12.8 & 16.3 \\
\hline 0.4 & -8.2 & 3.1 & 0.9 & 25.1 & 26.9 \\
\hline 0.5 & -4.4 & 4.8 & & & \\
\hline
\end{tabular}

The $\Delta E$ in eqn (2.13) can be obtained by first introducing values for $n_{1}^{\ddagger}$ and $n_{2}^{\ddagger}$ into eqn (2.14), solving the latter for $\Delta V$, and introducing this result into eqn (2.13). In table 1 the results of such a calculation are given choosing a rather large asymmetry: $\lambda_{1} / 4=12$ and $\lambda_{2} / 4=2$.

From table 1 one sees that the $\lambda / 4$ in eqn (2.6), namely $\Delta E$ at $\Delta V=0$, is 7.2. The latter is close to $\left(\lambda_{1}+\lambda_{2}\right) / 2$. The Brönsted slope $\partial \Delta E / \partial \Delta V$ for the system is seen from eqn (2.15) to be $n_{2}^{\ddagger}$. Thus, when the true slope is $0.1,0.3,0.6$ and 0.8 , say, one finds from the above $\lambda$ and the corresponding $\Delta V$ 's in table 1 that the slope calculated from eqn $(2.6)$ is $0.17,0.30,0.50$ and 0.72 , respectively, values which are fairly close to the true slopes.

\section{SOME DYNAMICAL ASPECTS OF LIGHT PARTICLE TRANSFER}

Chemical kinetics has received additional insight from recent studies with molecular beams, lasers, and infra-red chemiluminescence. ${ }^{6}$ On the theoretical side the main method for interpreting these data has involved computer-calculated classical 
trajectories of the atoms, ${ }^{6 b, 7}$ because of the difficulty of solving the fully threedimensional reactive collision problem numerically and quantum mechanically. Numerical quantum mechanical studies have been almost entirely confined to collinear collisions. $^{8}$ In the case of proton transfers no trajectory or quantum mechanical numerical studies appear to have been made as yet.

Some insight into the dynamics can be obtained by studying instead the transfer of a hydrogen atom between two heavier particles. The only quantum mechanical study which has appeared ${ }^{9}$ is that of a collinear collision between $\mathrm{HBr}$ and $\mathrm{Cl}$.

$$
\mathrm{Cl}+\mathrm{HBr} \rightarrow \mathrm{ClH}+\mathrm{Br},
$$

using a London-Eyring-Polanyi-Sato potential energy surface. This limitation of collinearity is perhaps not in itself too dismaying; the actual collisions in solution, with major steric or solvation features, can differ substantially from the usual threedimensional gas phase collisions.

The transmission probability was calculated for the reaction and, more specifically, for the formation of various vibrational states of the product $\mathrm{HCl}$ of this exothermic process. ${ }^{9}$ To analyze the results of this study and to obtain implications for other light particle transfer, Dr. Ellis of this laboratory has undertaken some classical trajectory studies on this and related systems. While the results will be described elsewhere, ${ }^{10}$ some features are summarized below.

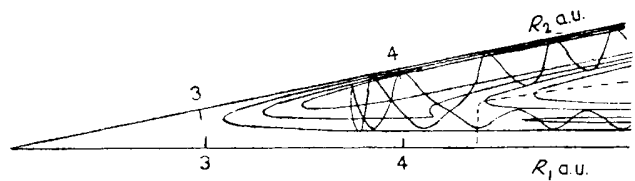

FIG. 1.-Skewed-axes plot of potential energy contours for reaction (3.1). $\quad R_{1}=R_{C l}-H, R_{2}=$ $R_{\mathrm{H}-\mathrm{Br}} / \mathrm{C}$, where $\mathrm{C}$ is the usual mass-scaling factor ${ }^{11}(0.987$ here). The dotted line denotes a transition state, and a reactive trajectory is also indicated.

A diagram of the surface used is given in fig. 1 in the usual skewed-axes form. ${ }^{11}$ (As is well known, plots in rectangular-axes form, while frequently used, are misleading for purposes of analyzing the dynamics of individual trajectories.) The radial coordinate is, essentially, a scaled $\mathrm{Cl} \cdots \mathrm{Br}$ distance, while the angular coordinate is the protonic coordinate. In one definition, the transition state is the line of steepest ascent from the saddle-point, indicated in fig. 1 by the dotted line. The latter is seen to be curved in the present highly exothermic instance.

A typical trajectory for reactants with an initial zero-point vibrational energy and with a substantial initial translational energy $(9 \mathrm{kcal} / \mathrm{mol}$ above the barrier height of $1 \mathrm{kcal} / \mathrm{mol}$ ) is indicated in fig. 1. For most of the trajectories corresponding to these and lower energies the relevant part of the dotted line is effectively perpendicular to the horizontal axis. Thereby, the reaction coordinate in this appreciably exothermic system is essentially the $\mathrm{Cl} \cdots \mathrm{Br}$ distance.

We found that the classical probabilities agreed approximately with the quantum mechanical values, for the transitions which were classically allowed, i.e., those for which the final vibrational states of products were attainable from the initial ones of reactants via real-valued classical mechanical trajectories. (Classically-forbidden transitions are those which require complex-valued trajectories. ${ }^{12}$ )

A substantial fraction of the trajectories which passed through the transition state region (i.e., across the dotted line) did or did not recross it to reform reactants, depending on the initial translational energy. The behaviour in the preliminary 
studies appears to suggest that a proper phasing of the $\mathrm{H}$-and $\mathrm{Cl}-\mathrm{Br}$ motions is needed for reaction. The recrossing itself "wastes" phase space. It implies that, apart from tunnelling corrections, the rate will be typically less than that predicted by transition state theory. ${ }^{13}$ However, even a factor of three as a discrepancy between transition state theory and the actual dynamics is a minor one, considering the large variations in rate which can be studied by variation of factors such as $\Delta G_{\mathbb{R}}^{\circ}$.

A second deduction can be made from the classical trajectories using semiclassical ${ }^{14}$ arguments : Because the zero-point energy of the vibrational motion (more precisely, a vibrational " action variable " $J$ ) is roughly constant up to the transition state region in the above study, the vibrational motion is substantially " adiabatic" 14 in this region of space. The "quantum number" of the vibration $N$ is related to $J$ by the well-known Bohr-Sommerfeld eqn (3.2) for a vibrational coordinate, a formula later justified by the WKB solution of the Schrödinger equation.

$$
J=\left(N+\frac{1}{2}\right) \boldsymbol{h} .
$$

While $N$ can have any real value classically but only integer values quantum mechanically, the same approximate adiabatic behaviour which led to a tendency to preserve $J$ classically in the present case, in the region up to the transition state, will lead to a similar tendency to preserve $N$ quantum mechanically in that spatial region. The vibrational energy is, for a harmonic oscillator of frequency $v$, equal to $J v$, both classically and quantum mechanically. Thus, apart from minor variations of $v$ in this region, the vibrational energy is also roughly constant. Since isotopic effects on the rate constant, in the absence of tunnelling, are largely attributed to differences in zero-point energies of reactants and the transition state, ${ }^{15}$ there should be essentially no isotopic effect on the rate constant in this appreciably exothermic system, when $\mathrm{H}$ is substituted for $\mathrm{D}$.

Finally, a type of Franck-Condon principle also operates in the region where the system moves from one channel to another, the momentum of the "slow" coordinate $\mathrm{Cl}-\mathrm{Br}$ being substantially conserved in that region. Here, the protonic motion is very nonadiabatic, and a significant increase of its vibrational action (and energy) occurs. Thus, in the reverse reaction vibrational energy should facilitate the proton transfer, an effect which might be observable in a suitably stabilized (e.g., intramolecularly hydrogen-bonded) system using short laser pulses.

In the case of the corresponding thermoneutral system

$$
\mathrm{Cl}+\mathrm{HCl} \rightarrow \mathrm{ClH}+\mathrm{Cl},
$$

the potential energy surface is quite different from that depicted in fig. 1 . The surface is now symmetrical about the bisector of the acute angle, and the dotted line representing the transition state now lies along that bisector. The reaction coordinate is, in the vicinity of the transition state, perpendicular (as before) to the dotted line and so now is substantially a motion of the proton. The original zero-point energy of the protonic motion has thus been lost, or really converted to motion along the reaction coordinate, when the system passes across the dotted line region. The full effect of an $\mathrm{H}$ and $\mathrm{D}$ isotopic difference in zero-point energy is thereby felt, yielding a maximum isotope effect (tunnelling corrections aside). These facts are wellknown, ${ }^{15}$ but it is interesting to see them borne out by the behaviour of the trajectories. The various dynamical results, classical and semiclassical, thus have implications for approximate dynamical treatments of light particle transfer, but we shall omit here further discussion of them.

The above remarks apply to potential energy surfaces such as that in fig. 1 and its analogues for less (or more) exothermic reactions. In the case of proton transfers 


\section{R. A. MARCUS}

in solution the effective surface is more apt to have potential energy wells in the two channels rather than free escape channels out to infinity, wells created by hydrogen bonding or by cage effects. Nevertheless, from semiclassical considerations, effects similar to those described above are expected to apply in this case also.

\section{WORK TERMS $w^{\mathrm{r}}$ AND $w^{\mathrm{p}}$}

The work term can be a composite of several terms. In the case of carbon acids or bases, which do not participate in hydrogen bonding, some desolvation of an attacking nitrogen oxygen base or acid may be needed ${ }^{2}$ and not compensated for by a favourable $\Delta G_{\mathbf{R}}^{\circ}$, and so contribute a term $w_{\mathrm{des}}^{\mathrm{r}}$ to $w^{\mathrm{r}}$. Again, in the large molecules which are usually involved and when the reactants are not joined by hydrogen bonding, an appreciable steric restriction may occur, and contribute a term $w_{\mathrm{st}}^{\mathrm{r}}$. For example, in the gas phase abstraction of a hydrogen atom from an alkane by a methyl group

$$
\mathrm{CH}_{3}+\mathrm{HR} \rightarrow \mathrm{CH}_{4}+\mathrm{R} \text {, }
$$

a steric factor of the order of $10^{-3}$ can be anticipated, ${ }^{16}$ and would correspond to a work term $w^{\mathrm{r}}$ of about $4 \mathrm{kcal} / \mathrm{mol}$. Such steric factors might be reduced somewhat by favourable $\Delta G_{R}^{\circ}$, but only a slight effect would be anticipated in the present case.

If one assigns to the partial desolvation a contribution of the order of $6 \mathrm{kcal} / \mathrm{mol}$ and assumes a steric effect of the above magnitude the net $w^{\mathrm{r}}$ for nonhydrogen bonded reactants would be about $10 \mathrm{kcal} / \mathrm{mol}$, which is of the same order as that needed to explain the data. ${ }^{2,17}$

Another contribution to the work term can also occur, when the immediate product of the third step in the reaction, eqn (2.4), is not the separated products but rather is a metastable intermediate which later ruptures, (cf. eqn (5.1) later). Whenever this last step has an activation barrier $w_{\mathrm{dec}}^{\mathrm{r}}$ which exceeds the barrier for the intermediate to reform the reactants, this $w_{\mathrm{dec}}^{\mathrm{r}}$ should in effect be added to the previously computed free energy barrier. We then have

$$
w^{\mathrm{r}}=w_{\mathrm{des}}^{\mathrm{r}}+w_{\mathrm{st}}^{\mathrm{r}}+w_{\mathrm{dec}}^{\mathrm{r}} \text {. }
$$

Of these $w^{\mathrm{r}}$ contributions, only the first two contribute to the $w^{\mathrm{r}}$ in eqn (2.7).

\section{NONEQUILIBRIUM SOLVENT POLARIZATION}

In electron transfer reactions a charge transfer occurs between two reactants, and the " charge centres" are usually some 5 to $10 \AA$ apart. In the transition state the electron cannot be in both places at the same time, and the solvent orientationvibrational polarization adopts a value which is some compromise. The solvent electronic polarization, on the other hand, can largely follow the motion of the electron being transferred. This situation, where the nuclear part of the solvent polarization is not that dictated by either charge centre alone and where the electronic part is dictated by the instantaneous position of the transferred electron and by the field due to the nuclear part, was termed " nonequilibrium polarization" and treated in some detail. 4,18

In the case of a simple proton transfer between two adjacent centres, as in eqn (2.3), the charge is transferred only over a relatively short distance, and an effect such as the above would be expected to be minor. In some cases, however, the assumed mechanism involves rearrangement of several bonds, with a somewhat larger displacement of charge in the proton transfer step (5.1). One example might be

$$
\mathrm{AH}+\mathrm{R}_{1} \mathrm{R}_{2} \mathrm{C}=\mathrm{N}^{+}=\mathrm{N}^{-} \rightarrow \mathrm{A}^{-}+\mathrm{R}_{1} \mathrm{R}_{2} \mathrm{CH}-\mathrm{N}^{+} \equiv \mathrm{N}
$$

(followed by elimination of $\mathrm{N}_{2}$ and by other processes). 
To obtain the potential energy of the transition state for any given configuration of the nuclei of the reaction complex and of the surrounding solvent, the Schrödinger equation is solved for the electronic wave function. When attention is focused on the electrons of the reactants, and the electrons of the solvent are treated, for reason of simplicity, as forming a polarizable dielectric continum, one obtains a nonlinear Schrödinger equation.

The free energy of formation of a nonequilibrium polarization state with an arbitrary orientation-vibration solvent polarization is given by ${ }^{19}$

$$
W_{\mathrm{rev}}=-\left[\left(1-1 / D_{\mathrm{op}}\right) / 8 \pi\right] \int \boldsymbol{D}^{2} \mathrm{~d} \boldsymbol{r}-\int \boldsymbol{P} \cdot \boldsymbol{D} \mathrm{d} \boldsymbol{r}+2 \pi c \int \boldsymbol{P}^{2} \mathrm{~d} \boldsymbol{r}
$$

neglecting dielectric image effects. $D(r)$ is the field directly due to the charges on the reactants, $1 / c$ is $1 / D_{\text {op }}-1 / D_{\mathrm{s}}, \boldsymbol{r}$ is any point in the solvent, $\boldsymbol{P}(\boldsymbol{r})$ is a function of the arbitrary orientation-vibration polarization, and $D_{\mathrm{op}}$ and $D_{\mathrm{s}}$ are the optical and static dielectric constants of the solvent, respectively. Ultimately, eqn (5.2) can be replaced by a more rigorous, statistical mechanical expression, but it will suffice for purposes of the present discussion.

The Schrödinger equation for the wave function $\psi$ of the electrons of the reactants, for any nuclear configuration $\boldsymbol{r}_{\mathbf{n}}$ of the reactants and (positions) of solvent molecules is obtained by minimizing ${ }^{20}$ the following functional $\mathscr{F}(\psi)$ with respect to $\psi$ at a given $\boldsymbol{P}$.

$$
\mathscr{F}(\psi)=\frac{\hbar^{2}}{2 \mu} \int|\nabla \psi|^{2} \mathrm{~d} \boldsymbol{r}_{1}+\int V\left(\boldsymbol{r}_{1}, \boldsymbol{r}_{\mathrm{n}}\right)|\psi|^{2} \mathrm{~d} \boldsymbol{r}_{1}+W_{\mathrm{rev}},
$$

where $\boldsymbol{r}_{1}$ denotes the totality of coordinates for the reactants' electrons, and $|\nabla \psi|^{2}$ really denotes a summation over such electrons $a, b, \ldots:\left|\nabla_{a} \psi\right|^{2}+\left|\nabla_{b} \psi\right|^{2}+\ldots$; $V\left(\boldsymbol{r}, \boldsymbol{r}_{\mathbf{n}}\right)$ includes the potential energy arising from interactions within the reactants and with the solvent molecules, apart from that included in the relatively long-range polarization term $W_{\text {rev }}$.

Ultimately, all values of the $r_{n}$ are considered and a suitable quantum and statistical mechanical average is made over $r_{\mathrm{n}}$. The $D$ appearing in eqn (5.2) is

$$
D(\boldsymbol{r})=-\nabla_{\mathrm{r}} \int|\psi|^{2} \mathrm{~d} \boldsymbol{r}_{1} /\left|\boldsymbol{r}-\boldsymbol{r}_{1}\right|,
$$

$1 /\left|\boldsymbol{r}-\boldsymbol{r}_{\mathbf{1}}\right|$ being an abbreviation for a sum over reactants' electrons, $1 /\left|\boldsymbol{r}-\boldsymbol{r}_{\mathbf{a}}\right|+$ $1 /\left|\boldsymbol{r}-\boldsymbol{r}_{\mathrm{b}}\right|+\ldots$

When the resulting (nonlinear) Schrödinger equation is solved for $\psi$, one obtains a $\psi$ which depends on $\boldsymbol{P}(\boldsymbol{r}) . \quad \mathscr{F}(\psi)$ then becomes a function of $\boldsymbol{P}$ which can then be obtained by then minimizing $\mathscr{F}$ with respect to $\boldsymbol{P}$. In the case of electron transfer reactions it was possible to introduce a simplifying approximation, writing $\psi$ as a linear contribution of two terms with weak overlap between them, one term being the same as for the reactants and the other being the same as for the products, and both reactants treated as spherical. ${ }^{18}$ The results obtained from eqn (5.2)-(5.4) can be shown (Appendix 1) to be equivalent to those obtained ${ }^{18}$ earlier by a different and in some respects less general method.

To the extent that the electronic wave function for the transition state of the reaction in eqn (5.1) could be similarly approximated for this purpose, ${ }^{21}$ the previous ${ }^{4,18}$ results for electron transfers could be adapted to that for proton transfer, and added to the contribution to $\Delta E$ in eqn (2.10). When $\psi$ cannot be written as a linear combination eqn (5.2)-(5.4) remain applicable but more formidable. Electronic structure calculations for the transition state of reactions such as (5.1) would therefore be helpful. 


\section{R. A. MARCUS}

When the electronic energy of the system has been obtained as a function of $\boldsymbol{r}_{\mathbf{n}}$ and $\boldsymbol{P}$, the latter remain to be treated, statistically as in transition state theory or dynamically. Examples of dynamical treatments for other or related potential energy surfaces are given in ref. (21) and (22).

\section{SUMMARY}

A substantial " reactant asymmetry" does not have a large effect on the slope of Brönsted plots (Section 2). Possible contributions to the work terms are summarized in Section 4, and the relation of the nonequilibrium polarization study in electron transfers to a possible one in proton transfer is considered in Section 5 . On the dynamics side, some results and implications of a recent study of dynamics of lightparticle transfer are described in Section 3.

\section{APPENDIX 1}

\section{RELATION OF EQN (5.3) TO THOSE IN REF. (18)}

If $\psi_{1}$ denotes the electronic wave function for the pair of reactants, as in ref. (18) and $\psi_{2}$ denotes that for the products, a trial $\psi$ is

$$
\psi=\sqrt{ } c_{1} \psi_{1}+\sqrt{ } c_{2} \psi_{2}
$$

This $\psi$ is introduced into eqn (5.3) and the variation, $\delta \mathscr{F}$, is calculated at fixed $\boldsymbol{P}$, and set equal to zero. The $\delta c_{1}$ and $\delta c_{2}$ are subject to

$$
c_{1}+c_{2}=1 \text {. }
$$

When the assumption of weak overlap of $\psi_{1}$ and $\psi_{2}$ is imposed, one can show that one obtains the result that the free energy of reactants with an arbitrary $\boldsymbol{P}$ equals that of the products, in this same $\boldsymbol{P}$ environment. This condition is identical with that imposed in ref. (18) to satisfy the Franck-Condon principle for these weak overlap systems. One next finds $\boldsymbol{P}$ by minimizing $\mathscr{F}$ subject to this new constraint, obtaining a relation the same as that used in ref. (18). The results in that paper are then obtained when the approximation of spherical reactants is introduced.

This work was supported in part by the Office of Naval Research.

${ }^{1}$ R. A. Marcus, J. Phys. Chem., 1968, 72, 891.

2 M. M. Kreevoy and D. E. Konasewich, Adv. Chem. Phys., 1971, 21, 243.

${ }^{3}$ G. W. Koeppl and A. J. Kresge, J.C.S. Chem. Comm., 1973, 371.

${ }^{4}$ R. A. Marcus, J. Chem. Phys., 1965, 43, 679.

${ }^{5}$ H. S. Johnston, Adv. Chem. Phys., 1960, 3, 131.

${ }^{6}$ E.g., (a) J. P. Toennies, Physical Chemistry, an Advanced Treatise, ed. H. Eyring, D. Henderson, and W. Jost (Academic Press, New York, 1974), vol. 6A, chap. 5; (b) articles in Faraday Disc Chem. Soc., 1973, 55, and ref. cited therein; (c) T. Carrington, MTP International Review of Science, Physical Chemistry, Series One, Chemical Kinetics, ed. J. C. Polanyi (Butterworths, London, 1972), vol. 9, p. 135 ; J. L. Kinsey, p. 173.

7 D. L. Bunker, Molecular Beams and Reaction Kinetics, ed. Ch. Schlier (Academic Press, New York, 1970), p. $355 \mathrm{ff}$; M. Karplus, p. $372 \mathrm{ff}$; J. C. Polanyi and J. L. Schreiber, Physical Chemistry, an Advanced Treatise, ed. H. Eyring, D. Henderson, and W. Jost (Academic Press, New York, 1974), vol. 6A, chap. 6.

8 E.g., E. M. Mortensen, J. Chem. Phys., 1968, 48, 4029; D. G. Truhlar, A. Kuppermann and J. T. Adams, J. Chem. Phys., 1973, 59, 395, and refs. cited therein; for 3-D calculations see A. Kuppermann and G. C. Schatz, J. Chem. Phys., 1975, 62, 2502, and A. B. Elkowitz and R. E. Wyatt, J. Chem. Phys., 62, 2504.

9 M. Baer, J. Chem. Phys., 1972, 62, 305.

$10 \mathrm{R}$. L. Ellis and R. A. Marcus, to be published.

11 S. Glasstone, K. J. Laidler and H. Eyring, The Theory of Rate Processes (McGraw-Hill, New York, 1941). 
12 J. Stine and R. A. Marcus, Chem. Phys. Letters, 1972, 15, 536; T. F. George and W. H. Miller, J. Chem. Phys., 1972, 56, 5668.

13 E. Wigner, Trans. Faraday Soc., 1938, 34, 29.

14 Cf., R. A. Marcus, Techniques of Chemistry, Investigation of Rates and Mechanisms of Reactions, ed. E. S. Lewis (John Wiley and Sons, New York, 1974), vol. 6, pt. 1, chap. 2 ; ref. (50) and (51) cited therein.

15 R. P. Bell, The Proton in Chemistry (Cornell University Press, Ithaca, New York, 2nd ed., 1973); R. P. Bell, Chem. Soc. Rev., 1974, 3, 513.

${ }^{16}$ E.g., J. A. Kerr, Free Radicals, ed. J. K. Kochi (John Wiley and Sons, New York, 1973), vol. 1 , chap. 1, p. 15 .

17 A. J. Kresge, S. G. Mylonakis, Y. Sato and V. P. Vitullo, J. Amer. Chem. Soc., 1971, 93, 6181 ; W. J. Albery, A. N. Campbell-Crawford, and J. S. Curran, J.C.S., Perkin II, 1972, 2206; M. M. Kreevoy and S. Oh, J. Amer. Chem. Soc., 1973, 95, 4805; A. J. Kresge, Chem. Soc. Rev., 1973, 2, 475.

18 R. A. Marcus, J. Chem. Phys., 1956, 24, 966, 979.

19 R. A. Marcus, J. Chem. Phys., 1965, 43, 3477, Appendix I.

${ }^{20} \mathrm{Cf}$. , S. I. Pekar, Untersuchungen über die Elektronentheorie der Kristalle (Akademie Verlag, Berlin, 1954).

${ }^{21}$ For a weak overlap approach, see also V. G. Levich, R. R. Dogonadze and A. M. Kuznetsov, Electrochim. Acta, 1968, 13, 1025 ; Electrokhym, 1967, 3, 739.

22 E.g., K. D. Godzik and A. Blumen, Phys. stat. sol., 1974, 66B, 569, and ref. cited therein; S. F. Fischer, G. L. Hofacker and M. A. Ratner, J. Chem. Phys., 1970, 52, 1934 ; S. F. Fischer and G. L. Hofacker, Internat. Symp. Phys. of Ice, Munich, 1968, eds. N. Richl, B. Bullemer and H. Engelhardt, (Plenum Press, New York, 1969), p. 369. 